\title{
THE ROLE OF THE DENTIST IN THE TREATMENT OF PATIENTS WITH TINNITUS
}

\author{
Marcin Wójcicki' , Jacek Szkutnik', Ingrid Różyło-Kalinowska² \\ 'Department of Functional Masticatory Disorders, Medical University of Lublin, Poland \\ ${ }^{2}$ Department of Dental and Maxillofacial Radiodiagnostics, Medical University of Lublin, Poland
}

\begin{abstract}
Tinnitus is a sensation of noise (such as ringing, roaring, buzzing, or whistling) without any external physical source of that sound. It affects about $15 \%$ of the adult population, of whom $2.5 \%$ report severely disturbing tinnitus. It is believed that temporomandibular disorders (TMD) are correlated with tinnitus. According to different studies, from $7 \%$ to $60 \%$ of patients with TMD also report tinnitus. The aim of this study was to point out the role of the dentist in managing patients with tinnitus. Untreated tinnitus can lead to serious debilitation and provoke suicidal tendencies. It is crucial to notice acute pulsating tinnitus, tinnitus with sudden, acute hearing loss, and acute post-traumatic tinnitus because these conditions are potentially life-threatening and require immediate referral to otolaryngologist. Worth noting is that similar psychological factors involved in the development of TMD (stress, depression, somatisation) also affect the generation of tinnitus. It is important to properly diagnose tinnitus and address it. Because of frequent coexistence of tinnitus and TMD, patients reporting to the dentist with TMD should be also asked about tinnitus. Different possible aetiologies and many factors involved in the onset and progression of tinnitus can impede the diagnosing process, which leads to the requirement of a multidisciplinary approach as well as discipline and cooperation on the part of the patient and the doctor.
\end{abstract}

KEY WORDS: treatment, tinnitus, temporomandibular disorders.

J Stoma 2019; 72, 2: 90-93

DOI: https://doi.org/10.5114/jos.2019.86989

\section{INTRODUCTION}

Clinical experience shows that tinnitus is more and more common among the symptoms reported by patients seeking treatment for temporomandibular disorders (TMD). Tinnitus is a sensation of noise (such as ringing, roaring, buzzing, or whistling) without any external physical source of that sound. It affects about 15\% of the adult population, of whom $2.5 \%$ report severely disturbing tinnitus [13]. It comprises a heterogenous group of conditions with different clinical presentations and aetiologies. The sound can be audible to an observer (objective tinnitus) but more frequently the sound can be only heard by the patient (subjective tinnitus). Tinnitus is usually a symptom rather than a disease by itself. However, when untreated it can debilitate the patient and lead to serious disorders such as depression. Tinnitus can also be associated with life-threatening pathologies like carotid stenosis or vestibular schwannoma. These characteristics mean that tinnitus should not be underestimated.

TMD is a wide term covering pain and dysfunction of masticatory muscles and the temporomandibular joints (TMJ). It is usually a complex of syndromes caused by multiple factors rather than a single condition. TMD has been proven to be correlated with tinnitus. It was reported that $60 \%$ of patients with tinnitus also had more

\section{JOURNAL OF} STOMATOLOGY CZASOPISMO STOMATOLOGICZNE

AdDRESS FOR CORRESPONDENCE: Marcin Wójcicki, Department of Functional Masticatory Disorders, Medical University of Lublin, 7 Karmelicka St., 20-400 Lublin, Poland, phone: +48730040194, e-mail: marcinow94@o2.pl 
than two symptoms of TMD (compared to $35 \%$ of patients in a tinnitus-free control group) and that among TMD patients from $7 \%$ to $60 \%$ had tinnitus, according to different studies [24]. Higher prevalence of tinnitus in TMD patients was also observed in other studies, supporting the hypothesis that TMD may play a causal role in the development of tinnitus $[2,16]$. However, it seems that there are no significant patterns of correlation with specific TMD [13].

The aim of this study was to point out the role of the dentist in managing patients with tinnitus.

\section{PATHOPHYSIOLOGY}

The mechanisms responsible for the development of tinnitus are still not well known, and there are many possible explanations. Objective tinnitus occurs when the sound is located within patient's body and it is possible to register it. The most common sources of this sound are vascular abnormalities (in this case, patients usually report heartbeat-synchronous pulsatile tinnitus), spontaneous contractions of muscles of the middle-ear or soft palate, and spontaneous otoacoustic emissions $[12,22]$. Although TMJ is not considered a typical factor in the development of objective tinnitus, there are reports of a possible causal connection between TMJ and objective tinnitus $[4,9,19,26]$.

Subjective tinnitus is a condition in which the patient hears sounds that do not exist. The sound is usually unformed in contrast to auditory hallucinations with perception of voices and melodies typical for some mental disorders. Tinnitus can originate from any lesions along the entire auditory pathway, but the most common are initial cochlear pathologies such as sudden hearing loss, noise trauma, presbycusis, hearing damage as a complication of systemic diseases (diabetes, hypertension, disorders of lipid metabolism, kidney disease) [8], damage caused by ototoxic drugs, infectious diseases including Lyme disease, syphilis, meningitis, and otitis media, or Meniere disease $[4,12]$. There are few concepts of subjective tinnitus pathophysiology. One of them suggests that the abnormal activity of the central nervous system occurs due to a loss of cochlear input by cochlear hair cell damage or some lesion of the auditory nerve causing deafferentation. This effect is analogous to the generation of phantom sensations after limb amputation. Another possible explanation is the effect of altered somatosensory input on auditory centre activity [20], which also fits with the observation that between $65.3 \%$ and $83.3 \%$ of patients can modulate their tinnitus by manipulations of the jaw, movements of the eyes, or pressure applied to the head and neck region involving TMJ [18]. It seems that modulation is increased in patients with somatic tinnitus, but some of the patients without an underlying somatic disorder are also able to modulate their tinnitus. Therefore, capability of tinnitus modulation cannot be used as a sole indicator for the somatic origin of tinnitus [18]. It seems also that any source of deep pain in the head and neck region can be involved in the development of tinnitus, such as dental pulpalgia [25]. It is also suspected that a tinnitus can develop due to the compression of the head of the mandible to the tympani chorda, auriculotemporal nerve, eustachian tube [5], and to the vascular bundle in the retrodiscal region containing the tympani artery and vein supplying the middle ear. Tinnitus can also be caused by penetration of inflammatory processes from the TMJ into the inner ear through the petrotympanic fissure [11], protective co-contraction of the tensor tympani muscle [17], and the impact of tensioning of the discomalleolar ligament (Pinto's ligament) [27]. The correlation between tinnitus and TMD could also emerge because of the common innervation by the mandibular branch of the trigeminal nerve of the masticatory muscles, tensor tympani muscle, and the tensor veli palatini muscle.

It is believed that psychological factors like emotions and stress are also associated with onset and persistence of tinnitus [12]. Vulnerability to tinnitus is increased by personality characteristics like hysteria, hypochondriasis, introversion, withdrawal, emotional isolation, and neuroticism [14]. Tinnitus is also strongly associated with depression [6].

\section{DIAGNOSIS AND TREATMENT}

The process of diagnosis should start from taking a detailed patient's history, making an assessment of the tinnitus severity, clinical ear examination, and audiological measurement of tinnitus and hearing function. In some cases, magnetic resonance imaging can also prove very helpful. This implies that a laryngologist should be the first specialist to diagnose the patient. It is worth mentioning here that acute pulsating tinnitus, tinnitus with sudden, acute hearing loss, acute post-traumatic tinnitus, and cases with suicidal tendencies require immediate diagnostics and treatment. After diagnosing certain types of tinnitus (tinnitus with hearing loss, with vertigo, with psychiatric comorbidity, post-traumatic tinnitus, or pulsatile tinnitus) the laryngologist should primarily focus on the treatment of disorders coexistent with tinnitus. When this treatment resolves the comorbidity but fails to improve the tinnitus the clinician should look for another possible source of the tinnitus. In rare cases of objective TMJ-related tinnitus the clinician should accurately address the cause. Possible source of this type of tinnitus can be herniation of the TMJ through the foramen of Hushke. In this case the treatment can be based on surgical closing of this foramen [19]. Another rare example can be objective tinnitus secondary to a fistula connecting the TMJ and the middle ear space, in which situation the treatment option is myringotomy [9]. After ruling out all possible otolaryngologic causes the doctor 


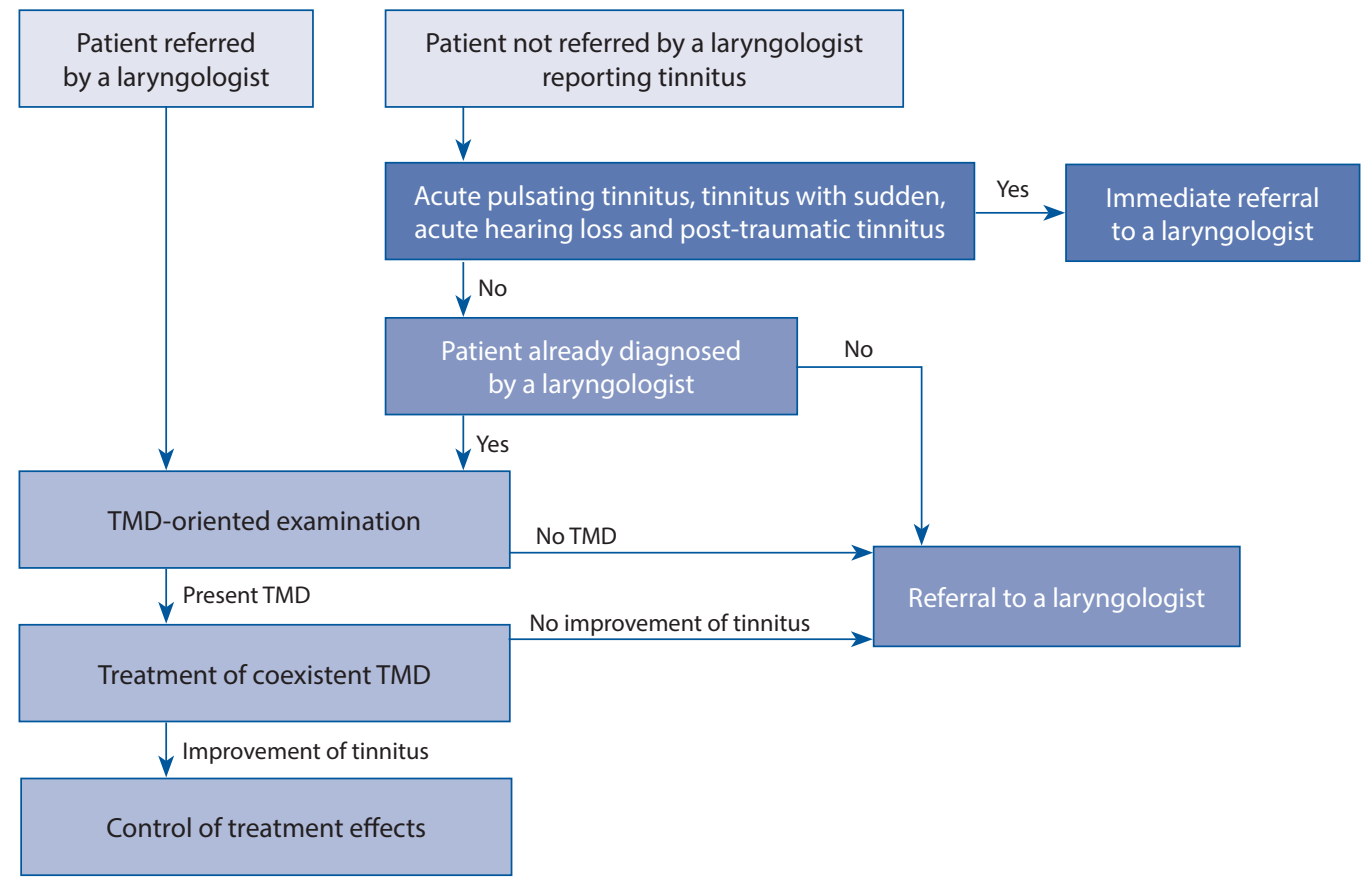

FIGURE 1. Guidelines for dentists for managing patients with tinnitus

should refer the patient to a dentist for further diagnostics and eventual treatment.

During examination of the patient the doctor should take into consideration any possible sources of deep pain and symptoms of TMD. If there is coexistent TMD and tinnitus, successful treatment of the former is likely to resolve or at least alleviate the patient's perception of tinnitus $[3,16,21,23,24]$. Selection of proper therapy should mainly depend on the coexistent TMD, maintaining the rule: "conservative therapy first". Patients with coexistent tinnitus with TMD should be treated by a dentist and physiotherapist [21]. The literature describes attempts at tinnitus treatment with physical therapy (manipulations, exercises, trigger point treatment) [15] and different occlusal appliances $[23,24,26]$, all with positive results. Tinnitus improved in 43\% [24] to 95\% [23] of patients. Moreover, the fluctuating tinnitus and acute or subacute tinnitus seem to be associated with better prognosis $[3,24]$. Dental treatment is not the last resort for the patient. If there is no coexisting TMD or if the TMD treatment failed to improve tinnitus, there are still available therapies focused mainly on tinnitus perception.

Psychological treatment are highly recommended. The most fundamental is counselling with psychoeducation, which grants the patient information and advice to better habituate to the tinnitus. It is an essential part of the management of every patient with tinnitus. Another possible treatment is cognitive-behavioural therapy. It is a well investigated psychotherapeutic strategy with clear evidence of improvement in the quality of the patient's life. It consists of psychoeducation, relaxation training, mindfulness-based training, attention-control techniques, imagery training, and exposure to difficult situations in order to modify maladaptive behaviour [12]. Also, tinnitus retraining therapy (combination of counselling and sound therapy) is a possible treatment option. It is supposed to make the patient unaware of their tinnitus unless they consciously focus on it. Significant improvements were seen in up to $80 \%$ of cases, but no well-controlled studies have been reported [10,22].

No pharmacological treatment can provide a replicable long-term reduction of tinnitus in excess of placebo effects. No drug has been approved by the US Food and Drug Administration or the European Medicines Agency for the treatment of tinnitus [1, 12]. However, some antidepressants (mainly tricyclic antidepressants) prescribed for coexistent depression disorders seem to lower the patient's perception of tinnitus. Also, benzodiazepines show some positive effects on tinnitus, but they cannot be administered for a prolonged time [22]. Carbamazepine may have a beneficial effect in rare subgroups of patients with typewriter tinnitus [12].

\section{CONCLUSIONS}

Tinnitus is usually a symptom of other diseases, some of these disorders are even life-threatening. However, sometimes it can become a disease by itself. Untreated tinnitus can lead to serious debilitation and provoke suicidal tendencies. It is worth noting that similar psychological factors involved in development of TMD (stress, depression, somatisation) [7] also affect the generation of tinnitus. Thus, it is very important to proper- 
ly diagnose tinnitus and address it. Because of frequent coexistence between tinnitus and TMD, patients reporting to the dentist with TMD should also be asked about tinnitus whilst taking their history. Different possible aetiologies and many factors involved in the onset and progression of tinnitus impede the diagnosing process, require a multidisciplinary approach, and demand discipline and cooperation on the part of the patient and the doctor. It is significant to diagnose and treat patients in the correct order. A simplified scheme of guidelines for a dentist is presented in Figure 1. Aan otolaryngologist should be the main doctor in the treatment of tinnitus. Patients with tinnitus that was discovered by a dentist should be referred for specialised otolaryngological treatment. After ruling out laryngological causes, patients should be referred to a dentist for TMD diagnostics and treatment. When there is no TMD or the TMD treatment did not alleviate the perception of tinnitus the patient should be referred back to the otolaryngologist for tinnitus-oriented treatment, such as tinnitus retraining therapy or cognitive-behavioural therapy. It is especially important to notice acute pulsating tinnitus, tinnitus with sudden, acute hearing loss, and acute post-traumatic tinnitus, because these conditions are potentially life-threatening and require immediate referral to an otolaryngologist.

\section{CONFLICT OF INTEREST}

The authors declare no potential conflicts of interest with respect to the research, authorship, and/or publication of this article.

\section{References}

1. Baguley D, McFerran D, Hall D. Tinnitus. Lancet 2013; 382: 16001607.

2. Bousema EJ, Koops EA, van Dijk P, Dijkstra PU. Association between subjective tinnitus and cervical spine or temporomandibular disorders: a systematic review. Trends Hear 2018; 22: 1-15.

3. Buergers R, Kleinjung T, Behr M, Vielsmeier V. Is there a link between tinnitus and temporomandibular disorders? J Prosthet Dent 2014; 111: 222-227.

4. Çakur B, Yaşa Y. Correlation between tinnitus and petrotympanic fissure status among patients with temporomandibular joint dysfunction. J Oral Maxillofac Surg 2016; 74: 47-52.

5. Camparis CM, Formigoni G, Teixeira MJ, Siqueira JTT. Clinical evaluation of tinnitus in patients with sleep bruxism: prevalence and characteristics. J Oral Rehabil 2005; 32: 808-814.

6. Fernandes G, Goncalves DA de G, Siqueira JTT de, Camparis CM. Painful temporomandibular disorders, self reported tinnitus, and depression are highly associated. Arq Neuropsiquiatr 2013; 71: 943-947.

7. Fillingim RB, Ohrbach R, Greenspan JD, et al. Psychological factors associated with development of TMD: the OPPERA prospective cohort study. J Pain 2013; 14: T75-T90.

8. Guzek WJ, Sułkowski WJ, Kowalska S, Makowska Z. Tinnitus Center at the Nofer Institute of Occupational Medicine - earliest experience. Medycyna Pracy 2002; 53: 461-464.

9. Hidaka H, Tatewaki Y, Sakamoto M, et al. Transient hearing loss and objective tinnitus induced by mouth opening: a rare connec- tion between the temporomandibular joint and middle ear space. J Laryngol Otol 2016; 130 (Suppl 3): S173.

10. Jastreboff PJ. 25 years of tinnitus retraining therapy. HNO 2015; 63: 307-311.

11. Kozak M, Chruściel-Nogalska M, Ey-Chmielewska H. Tinnitus, diagnosis and therapy based on selected literature - preliminary report. Dent Forum 2014; 42: 85-88.

12. Langguth B, Kreuzer PM, Kleinjung T, De Ridder D. Tinnitus: causes and clinical management. Lancet Neurol 2013; 12: 920-930.

13. Manfredini D, Olivo M, Ferronato G, Marchese R, Martini A, Guarda-Nardini L. Prevalence of tinnitus in patients with different temporomandibular disorders symptoms. Int Tinnitus J 2015; 19: 47-51.

14. McCormack A, Edmondson-Jones M, Fortnum H, et al. The prevalence of tinnitus and the relationship with neuroticism in a middle-aged UK population. J Psychosom Res 2014; 76: 56-60.

15. Michiels S, Naessens S, Van de Heyning P, et al. The effect of physical therapy treatment in patients with subjective tinnitus: a systematic review. Front Neurosci 2016; 10: 545.

16. Mottaghi A, Menéndez-Díaz I, Cobo JL, González-Serrano J, Cobo T. Is there a higher prevalence of tinnitus in patients with temporomandibular disorders? A systematic review and metaanalysis. J Oral Rehabil 2019; 46: 76-86.

17. Okeson JP. Signs and symptoms of temporomandibular disorders. In: Management of temporomandibular disorders and occlusion. $7^{\text {th }}$ ed. Elsevier, St. Louis $2013 ; 129-160$.

18. Ralli M, Greco A, Boccassini A, et al. Subtyping patients with somatic tinnitus: modulation of tinnitus and history for somatic dysfunction help identify tinnitus patients with temporomandibular joint disorders. PLoS One 2018; 13: e0202050.

19. Ryu KH, Baek HJ, Hur DG. Spontaneous temporomandibular joint herniation into the external auditory canal through a patent foramen of Huschke: a case report. Ann Med Surg 2017; 18: 33-35.

20. Shore S, Zhou J, Koehler S. Neural mechanisms underlying somatic tinnitus. Prog Brain Res 2007; 166: 107-123.

21. Skog C, Fjellner J, Ekberg E, Häggman-Henrikson B. Tinnitus as a comorbidity to temporomandibular disorders - a systematic review. J Oral Rehabil 2019; 46: 87-99.

22. Swain SK, Nayak S, Ravan JR, Sahu MC. Tinnitus and its current treatment - still an enigma in medicine. J Formos Med Assoc 2016; 115: 139-144.

23. Taylor LP, Sletten WO, Dumont TD. The effect of specially designed and managed occlusal devices on patient symptoms of tinnitus: a cohort study. Cranio 2019; 37: 101-110.

24. Tullberg M, Ernberg M. Long-term effect on tinnitus by treatment of temporomandibular disorders: a two-year follow-up by questionnaire. Acta Odontol Scand 2006; 64: 89-96.

25. Wright EF, Gullickson C. Dental pulpalgia contributing to bilateral preaurieular pain and tinnitus. 1996; 10: 166-168.

26. Wright EF, Syms CA, Bifano SL. Tinnitus, dizziness, and nonotologic otalgia improvement through temporomandibular disorder therapy. Mil Med 2000; 165: 733-736.

27. Wysocki J, Krasucki KP, Szałwiński M, Powała J, Jędrych E, Kotlarski M. Clinical aspect of discomalleolar ligament morphology. Otorynolaryngologia 2009; 8: 66-71. 
